\title{
Nintento Wii as an educational implement. Reality or fiction?
}

\author{
Sebastián López-Serrano ${ }^{1}$, Sara Suárez-Manzano ${ }^{1}$, Alberto Ruiz-Ariza ${ }^{1 \mathrm{a}}$, and Emilio J. \\ Martínez-López ${ }^{1}$ \\ ${ }^{1}$ Faculty of Humanities and Education Sciences, University of Jaen, Campus Las Lagunillas s/n, Jaen, \\ Spain
}

\begin{abstract}
The current society is in constant evolution in search of new methodologies that allow an integral formation of the students. The current scientific evidence has corroborated that one way to stimulate cognitive function is through the usual practice of physical activity (PA). It has been shown that the inclusion of play activities such as exergames (EX) during the academic day, helps to increase the amount of daily PA, as well as to improve socialization and cognitive performance. The aim of this research is to review if PA practice through the use of the Nintendo Wii console positively influences on cognition of children and adolescents. In conclusion, the practice of EX, and specifically the Nintendo Wii, can be key in the improvement of cognitive and academic performance. Keywords: Nintendo Wii, cognitive performance, socialization.
\end{abstract}

\section{Introduction}

Recent studies have shown that the systematic practice of Physical Activity (PA) can contribute to the improvement of Cognitive Performance (CP) in children [1] and adolescents [2]. PA produces physiological adaptations such as increased blood flow by increasing levels of brain-derived neurotrophic factor (BDNF) that promotes neuron growth and survival by improving learning ability [3]. In addition, the practice of PA helps to reduce the high levels of sedentary that we are currently a great resource for improving health, disease prevention or increased life expectancy [4].

In relation to the above, active video games like the Nintendo Wii, appear as a key opportunity to increase the daily amount of PA in a playful way. These active video games present great popularity among young people, and their interactive dynamics generates a connection between the player and the game. In this way, the body movements are interpreted reflecting the activity carried out during its development in the device. In addition, this type of video game mode favors peer social relations [5] and increases caloric expenditure [6]. On the other hand, the potential that the Exergames (EX) present in the

\footnotetext{
${ }^{\text {a }}$ Corresponding author: arariza@ujaen.es
} 
transformation of sedentary time into physically active time is known [7]. Therefore, knowing the relation of the use of the EX in the educational framework could favor to increase the levels of PA, the reduction of the sedentary time that children currently spend in the classroom [8] and favor their personal and social development, with direct consequences on school performance.

\section{Objective}

To carry out a current bibliographic review that associates the effect of the Nintendo Wii with the academic performance in children and adolescents.

\section{Sample}

Four interventional studies were included. The total sample of articles analysed ranged from 6 to 19 years. The total number of participants was 252 children and adolescents.

\section{Methodology}

Two specialized databases (SportDiscus and Web of Science) were used and the search was limited to the last 7 years. The search terms used can be seen in Table 1. The search was conducted in English.

Table 1. Search strategies in databases.

\begin{tabular}{ll}
\hline SportDiscus & $\begin{array}{l}\text { Topic: (Exergames OR Nintendo Wii) AND Topic: (cognitive OR academic } \\
\text { performance) AND Topic: (Children OR adolescent) }\end{array}$ \\
\hline Web of Science & $\begin{array}{l}\text { TX (Exergames OR Nintendo Wii) AND TX (Cognitive OR academic } \\
\text { performance) AND TX (children OR adolescent) }\end{array}$ \\
\hline
\end{tabular}

The selection criteria used were:

$1^{\circ}$ ) The study was performed with a healthy population.

$2^{\circ}$ ) The population outside children and adolescents between 6 and 18 years.

$3^{\circ}$ ) The EX and the variable of academic performance were clearly described.

\section{Results}

The flow of results obtained after the completion of the search is shown in Figure 1. After elimination of duplicates, excluded by title or age, a total of 126 articles were reviewed. These studies followed the review criteria discussed above. Finally, 4 articles were included for analysis. 


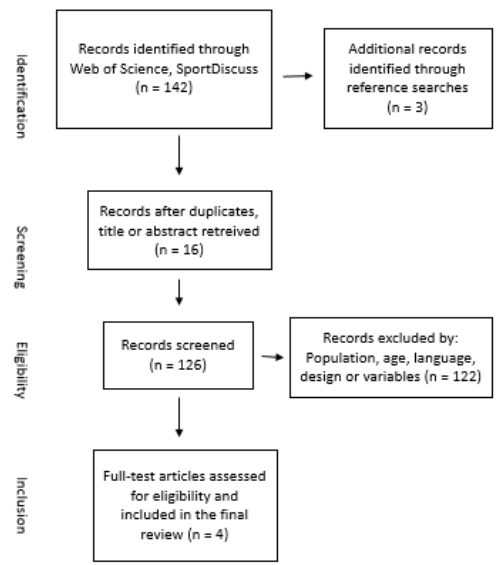

Fig. 1. Flow of articles through the search process.

The four studies selected were intervention studies [9-11]. 2 studies conducted the groups randomly $[11,12]$ and 2 did not randomize groups $[9,10]$. They present a total of 252 subjects. All of them belong to the American continent (USA). The PA variables that were controlled were the heart rate [9], strength [11], the score given by the game after the practice session [10] and the time to go one mile before and after the intervention with the Nintendo Wii [10]. Performance measures at the cognitive level focused on executive functions. These studies included covariates, including socio-educational level of families, BMI, sex and age.

The studies showed a positive relationship between the PA level performed during EX and cognition. Best [9] demonstrated that with an EX session they improved the cognitive functions of children. Staiano et al. [11], after ten weeks of intervention, indicated that young people who practiced EX competitively and cooperatively improved their executive functions more than the group that did not perform any type of activity through these devices. On the other hand, Flynn et al. [12], showed that children who played for five weeks at EX improved their cognitive performance, and as they played more, they gained greater benefits. Gao et al. [10], following a six-week intervention, concluded that children undergoing EX practice performed better in terms of executive function and effort.

\section{Discussion}

The results of this review are related to the majority of previous research that relates a positive association between the play of any modality of EX and the improvement of cognition [8]. These studies showed benefits in cognitive aspects -attention, understanding and motivation- as academic achievement. Other studies focused on the benefits of active video games on health $[6,13]$, positively influencing an increase in caloric expenditure and a better level of fitness. On the other hand, information regarding the amount and intensity of exercise that is necessary to positively influence cognition is very scarce. Analyzing the degree of intensity (low / high) as a predictor of a better or worse cognitive response is the most appropriate, it would help the integration of this type of activities for its development in schools. 


\section{Conclusion}

In conclusion, the use of the Nintendo Wii presents great potential for the development of activities during the school day. The PA that is needed during the development of this video game favors an improvement of the cognition in the children and adolescents. Therefore, the inclusion of this type of activities in the educational framework would help a greater motivation on the part of the students, having a positive effect on their academic performance. In addition, it highlights its social factor, benefiting the relationships between peers and teachers, as well as the health benefits associated with the practice of PA itself.

\section{References}

1. E.A. Haapala, Journal of human kinetics, 1, 55 (2013)

2. D.N. Ardoy, J.M. Fernández-Rodríguez, D. Jiménez-Pavón, R. Castillo, J.R. Ruiz, F.B. Ortega, Scandinavian Journal of Medicine \& Science in Sports, 1, 52 (2014)

3. L. Chaddock, Heyman, C.H. Hillman, N.J. Cohen, A.F. Kramer, Monographs of the Society for Research in Child Development, 4, 25 (2014)

4. World Health Organization (2015). Extracted on January 2, 2016 in http://www.who.int/topics/physical_activity/es/

5. V.M. Nurkkala, J. Kalermo, T. Jarvilehto, Journal of Communication and Computer, 11, 403 (2014)

6. N. Dutta, M.A. Pereira, Journal of Physical Activity and Health, 6, 890 (2015)

7. A.G. LeBlanc, J.P. Chaput, Preventive Medicine (2016)

8. E. Norris, M. Hamer, E. Stamatakis, The Journal of Pediatrics, 172, 40 (2016)

9. J.R. Best, Developmental psychology, 5, 1501 (2012)

10. Z. Gao, J.E. Lee, Z. Pope, D. Zhang, Games for Health Journal, 5, 318 (2016)

11. A.E. Staiano, A.A. Abraham, S.L. Calvert, Developmental psychology, 2, 337 (2012)

12. R.M. Flynn, R.A. Richert, A.E. Staiano, E. Wartella, S.L. Journal of educational and developmental psychology, 1, 209 (2014)

13. Z. Gao, S. Chen, D. Pasco, Z. Pope, Obesity reviews, 9, 783 (2015) 